\title{
Spring Migration Notes of The Chicago Area
}

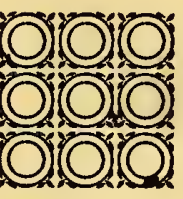

Compiled by James D. Watson George Porter Lewis Nathan F.Leopold, Jr. 



\title{
Spring Migration Notes \\ of \\ The Chicago Area
}

\author{
Compiled by \\ James D. Watson \\ George Porter Lewis \\ Nathan F. Leopold, Jr.
}


Digitized by the Internet Archive in 2015

https://archive.org/details/springmigrationn00wats 
NATHAN F. LEOPOLD, JR.

4754 GREENWOOD AVENUE

CHICAGO, ILLINOIS

October 22, 1920.

Mr. Ruthren Dease,

$1222 N$. State St.

Chiago, III.

Dear Sir,

Ar in receipt of your valued comrunication of the 21 t inst, and take great pleasure in enclosing, under separate cover, a copy of the pamphlet you desire. As to your kind offer to make application for membershing in the A.O.U. for ny to comorkers, I beg to state that unfortunately I have already made application for them. Thanking you for your kindness, I remain,

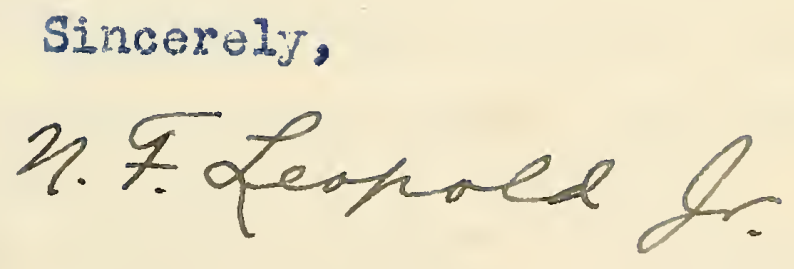





\section{Introduction}

7 HE following list of migration dates has been compiled entirely from the lists of Messrs. Locke 1. Mackenzie, Sydney Stein and ourselves. These lists cover the spring migration for the eight years 1913-1920. and include the earliest date for each bird each year.

All specimens were either taken or clearly seen, and the list should contain some valuable dates. No bird, not actually seen by one of the above mentioned has been included.

By the words Chicago Area, we mean that portion of the country lying within a radius of thirty miles from the city itself. This region includes several excellent stations for observation, such as Miller, ,Ind., Hinsdale, Argo, Riverside, Highland Park, and Hyde Lake, Ill., and the city parks, a diversified region containing lakes, woods, swamps, and rivers. The list contains two hundred and twenty-seven species seen in this area, five seen only in the fall migration, and five sub-species whose occurrence in our area is doubtful. This totals two hundred and thirty-seven species and sub-species. The names used are those given in the $1910 \mathrm{~A}$. O. U. check list.

We wish to express our sincere thanks and gratitude to Messrs. H. K. Coale, Locke Mackenzie, and Sydney Stein, whose kind co-operation has made the work possible.

THE AUTHORS. 



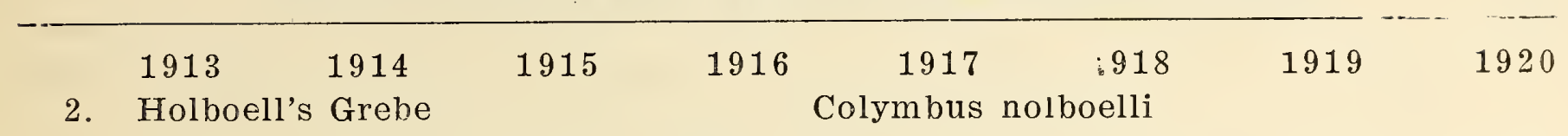

Mar. 20

Rare visitant; winters on Lake Michigan. One bird remained in the park lagoons several days.

3. Horned Grebe

Colymbus auritus

Apr. 13

Apr. 8 Apr. $9 \quad$ Mar. 24 Apr. $5 \quad$ Apr. 28

Common migrant.

6. Pied-billed Grebe Podilymbus podiceps

Mar. 30 Mar. 26 Apr. 2 Mar. 25 Mar. 26 Mar. 15 Apr. 5 Mar. 30 Common summer resident; most common during migrations.

7. Loon

Gavia immer

Apr. 7 Mar. 16 Apr. 1 Apr. 7

Fairly common migrant.

11. Red-throated Loon

Gavia stellata

Feb. 21

Very rare winter visitant. One, apparently wounded observed in Jackson Park, Feb. 21, 1915. Woodruff reports three dead birds at Evanston.

51. Herring Gull

Larus argentatus

Jan. 1 Jan. 1 Jan. 1 Jan. 2 Jan. 1 Jan. 1 Jan. 1 Jan. 1 Abundant winter resident: a few non-breeding birds remain during the summer.

54. Ring-billed Gull

Larus delawarensis

Apr. 26 Jan. 2 Jan. 11 Jan. 2 Apr. $6 \quad$ Jan. 18 Mar. $14 \quad$ Jan. 1

A fairly common, but erratic visitant.

60. Bonaparte's Gull Larus philadelphia

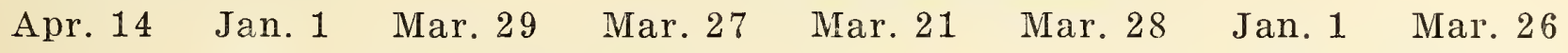
Common migrant, sometimes wintering. A few non-breeding birds remain during the summer.

69. Forster's Tern

Sterna forsteri

Apr. $4 \quad$........... May 2

May 3

Uncommon migrant, but probably more common than generally supposed, especially in the fall at Miller.

70. Common Terı

Sterna hirundo

Apr. 30 Apr. 25 Apr. 26 May 2 Apr. 21 Apr. 17 May 9 Apr. 20 Common migrant.

77. Black Tern

May 24 Apr. 21 May 9

Hydrochelidon nigra surinamensis

Breeds commonly in the swamps and small lakes in the vicinity.

129. Merganser

Mergus americanus

Apr. 2 Mar. 9 Jan.18 Jan. $2 \quad$ Feb.19 Jan.26 Jan. 1 Jan. 1 Common winter resident.

130. Red-breasted Merganser

Mergus serrator

Mar.30 Mar.30 Mar.25 Jall.23 Mar.31 Apr.13 Mar.18 .Mal.18 Abundant migrant.

131 Hooded Merganser

Lophodytes cucullatus

Apr. 21 Mar. 1

Rare summer resident.

Apr. 5 Mar. 26 
1913

1914

1915

132. Mallard

\section{Jan. 1 Mar. 22 May}

1916

1917

1918

1919

1920

Anas platyrhynchos Fairly common summer resident; occasionally winters.

137. Baldpate

Mareca americana
Apr. 22 Apr.
Mar. 30 Mar. 31
Apr. 20
Formerly a common migrant; now apparently becoming rare.

Mar. 18

139. Green-winged Teal

Nettion carolinense

Apr. $15 \quad$ Mar.27

Mar. 11

Uncommon migrant.

140. Blue-winged Teal

Apr. 20 Apr. 2

Querquedula discors

Summer resident.

142. Shoveller

Apr. 11 Apr. 5

Apr. 22 Mar. 24 Apr. 2

Apr. 12 Apr. 10

Spatula clypeata

Apr. 7 Apr. 25 Apr. 12 Apr. 24

Fairly common migrant.

143. Pintail

Apr. 15

Dafila acuta

Apr. 7 May 11 Apr. 12 Apr. 17

Fairly common migrant.

144. Wood Duck

Feb. 18

Aix sponsa

Mar. $1 \delta$

Rare summer resident.

146. Redhead

Mar. 22

Marila americana

Mar. 30

Mar. 21

Fairly common, but erratic migrant.

14.7. Canvasback

Marila valisineria

Mar. 1

Apr. 10

Formerly common migrant, but becoming rare.

149. Lesser Scaup Duck Marila affinis

Mar. 30 Mar. 19 Feb. 15 Mar. 12 Mar. 25 Mar. 24 Jan. 1 Mar. 22

Abundant migrant sometimes wintering.

150. Ring-necked Duck Marila collaris

Mar. 30 Mar. 28 Mar. 12 Mar. 31

Apr. 12 Apr. 24

Uncommon migrant.

151. Goldeneye Clangula clangula americana

Mar. 30 Mar. 19 Jan. 1 Jan. 2 Feb. 1 Mar. 11 Jan. 1 Jan. 1 Very common winter resident.

152. Barrow's Goldeneye Clangula islandica

Jan. 13

Very rare winter visitant. Taken once in the area, by Woodruff. A flock of six wintered in 1919-1920.

153. Bufflehead

Mar. 31 Apr. 1

Fairly common migrant.

154. Oldsquaw Common winter resident.
Charitonetta albeola

Apr. 1 Mar. 11 Mar. 7 Mar. 25

Harelda hyemalis

Jan. 5 Jan. 17 Feb. 29 Feb. $1 \quad$ Feb. 22

Jan: 11 


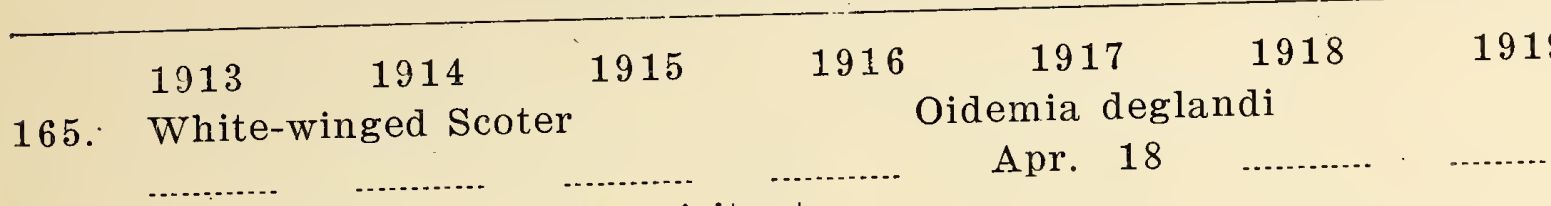

Uncommon winter visitant.

167. Ruddy Duck

\section{Apr. 15 Apr. 16}

Not uncommon migrant.

171a. White-fronted Goose
Erismatura jamaicensis

$$
\text { Apr. } 9
$$

Apr. 17

\section{Anser albifrons gambeli}

$$
\text { Apr. } 7
$$

Rare migrant. A small flock seen in Hinsdale swamp by Mr. Watson.

172. Canada Goose

Branta canadensis canadensis

Feb. 23 Jan. 25 Mar. 8 Jan. 3 Mar. 10. Feb. 10 Mar. 25 Mar. 3 Common migrant often wintering; spends the day on the edge of the ice on the lake, and the night inland on some cornfield of the previous season

150. Bittern Botaurus lentiginosus
Apr. 2
Mar. 2
Apr. 11 Apr. 24 May 4
Apr. 7 Apr. 22

Common summer resident.

191. Least Bittern Ixobrychus exilis

May 1 Apr. 27 May 8 June 8 May $30 \quad$ May 10 May 15 Fairly common summer resident.

194. Great Blue Feron

May 11

9 Mar. 31

Apr. 29

Ardea herodias herodias

Not uncommon summer resident.

200. Little Blue Feron

Florida caerulea

$$
\text { May } 8
$$

Casual visitant. One observed in Jackson Park, May 8, 1915.

201. Green Heron .

Butorides virescens virescens

Apr. 22 Apr. 15 Apr. 22 Apr. 16 Apr. 18 Apr. 28 Apr. 19

Fairly common summer resident; common during migration.

202. Black-crowned Night Heron Nycticorax nycticorax nacvius.

Mar. 29 Apr. 20 Apr. 18

Mar. 31 Apr. 28

May 10

Rather common summer resident.

203. Yellow-crowned Night Heron

Nyctanassa violacea

Apr. 14

Accidental straggler in the area. One, observed in Jackson Park, April 14, 1918. The bird showed no signs of having been in captivity, and flew well.

206. Sandhill Crane

$$
\text { May } 2
$$

Grus mexicana

Mar. 24

Apr. 22

Formerly common, but now extremely rare migrant. One seen in Jackson Park, attempting to fiy against a strong wind. It continued its efforst for half an hour but was finally blown out of sight to the south.

208. King Rail.

Rallus elegans

Apr. 19 Apr. 24 Apr. 25 May 20

May 4 Common summer resident.

212. Virginia Rail.

Rallus virginianus

Apr. 22 Apr. 25 Apr. 18 Apr. 25 Apr. 18 May 5 May 10 Apr. 24 Common summer resident. 


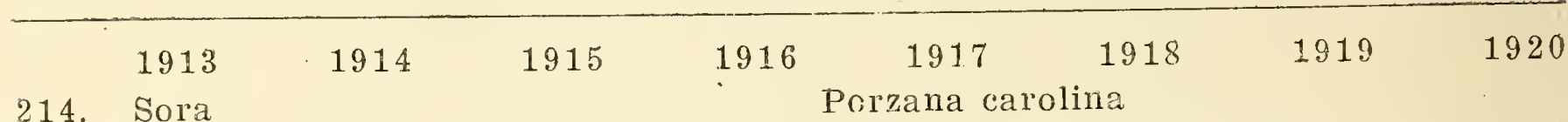

214. Sora Apr. 25 Apr. 24 Apr. $25 \quad$ Apr. 20 May 11 May 10 Apr. 24 Common summer resident.

219 Florida Gallinule Gallinula galeata
Apr. 28 May 8 May 20 May 11
May 15

Fairly common summer resident.

221. Coot

Fulica americana

Mar. 31 Feb. 28 Mar. 21 Mar. 18 Mar. 23 Mar. 10 Mar. 31 Mar. 14 Common summer resident.

224. Wilson's Phalarope

Steganopus tricolor

May 9 May 20

Nests in the Calumet region.

228. Woodcock

Philohela minor

Apr. 4: May 26 Mar. 25 Mar. 23 May 11 May 17 May 12 Not uncommon summer resident.

230. Wilson's Snipe

Gallinago delicata

Mar. 29 Apr. 10 Apr. 7 Apr. 16 Mar. 24 Apr. 13 May 4 Mar. 31

Common migrant.

232. Long-billed Dowitcher

Macroramphus griseus scolopaceus

May 14

Very rare migrant; about seven seen at Calumet May 14, 1920, of which one, a fine adult female was taken, and is now in the collection of $\mathrm{N}$. F. Leopold.

239. Pectoral Sandpiper

Piscbia maculata

Apr. 18 Apr. 19 Apr. 5

Mar. 26

May 8

Common migrant.

242. Least Sandpiper

Pisobia minutilla

May 19

May $20 \quad$ May 13

Common migrant.

243a. Red-backed Sandpiper

Pelidna alpina sakhalina

May 26

Not uncommon migrant. A flock of six was seen at Jackson Park, in

October, 1916.

246. Semipalmated Sandpiper

Ereunetes pusillus

May 24

Common migrant, seen more often in fall than in spring.

248. Sanderling

Calidris leucophaea

May 22

May 24

Common migrant, seen more often in August, September and October.

254. Greater Yellowlegs

Totanus melanoleucus

May 3 Apr. 22 Apr. 29 Apr. 11

Common migrant, but less common than the succeeding species.

255. Yellowlegs

Totanus flavipes

Apr. 28 Apr. 21 May $6 \quad$ Apr. 26 May 6

May 14 Common migrant. 
SPRING MIGRATION NOTES OF THE CHICAGO AREA

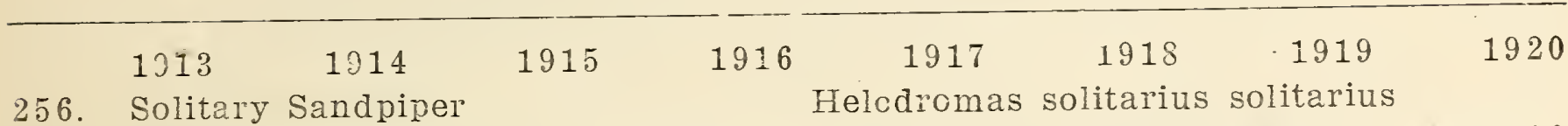

Apr. 26 Apr. 26 Apr. 19 Apr. 20 Apr. 18 May 11 May 3 May 10 Common migrant.

263. Spctted Sandpiper Actitis macularia

Apr. 22 Apr. 18 Apr. 2 Apr. 10 Apr. 24 Apr. 28 Apr. 22 Apr. 24 Ccinmon summer rezident.

272. Golden Plover Charadrius dominicus dominicus

$$
\text { May } 22 \quad \text {.................... Mà } 30
$$

Ccmmon migrant in fall, less common in spring.
273. Killdeer

Mar. 20 Mar. 14 Mar. 12 Mar. 18 Mar. 10 Mar. 12 Mar. 16 Mar. 13 Common summer resident.

274. Semipalmated Plover Aegialitis semipalmata Nay 24

Rather common migrant, especially in the fall.

277. Piping Flover

Aegialitis meloda

Rather rare summer resident. Nests at Miller and at Beach.

283a. Ruddy Turnstone

Arenaria interpies morinelia.

$$
\text { Míy } 24
$$

Nct uncommon fall migrant: rarely seen in spring.

289. Bobwhite

Colinus virginianus virginianus

Mar. 8 Mar. 29 Jan. 1

Formerly e y common penment resident, but now ratier rare.

305. Prairie Chicken

Tympanuchus americanus americanus

$$
\text { Apr. } 28 \text { Apr. } 18
$$$$
\text { Apr. } 15
$$

Formeriy abundant permanent resident; now rather rare.

*** Ring-neaked Pheasant

Nisr. 29

Phasianus torquatus

ntroduced into the area, but without great success. The bird reccrded

mey hare escared from cne of the game farms in the vicinity. No authentic record of its breeding in our area.

316. Mourning Dove

Zenaidura macroura carolinensis

Apr. 2 Apr. 5 Apr. 8 Apr. 4 Mar. 23 Apr. 27 Mar. 25 Apr. $2:$

Fairly commen summer resident.

325. Turkey Vulture

Cathartes aura septentrionalis

Apr. 10

Nar. 31

Rəre visitant from the south.

331. Narsh Hawk Circus hudsonius

Fè. 16 Mar. 14 Mar.27 Mar. 18 Mar. 10 Apr. 13 Mar. 19 Mar. I5 Common summer resident.

332. Sharp-shinned Hawk Accipiter velox

Apr. 10 Apr. 9 Mar. 23 Mar. 15 Feb. 14 Apr. 7 Mar. 15 Common summer resident.

333. Ccoper's Hawk Accipiter cooperi

Maq. 30 Apr. 18 Apr. 9 Apr. 13 Mar. 24. Nay 3 Apr. 10 Apr. 1 Common summer resident. 


$\begin{array}{llcccc}1913 & 1914 & 1915 & 1916 & 1917 & 1918 \\ \text { Red-tailed } & \text { Hawk } & & & \text { Buteo borealis } & \text { borealis }\end{array}$

337. Red-tailed Hawk

Mar. 30 Feb. $9 \quad$ Mar. 18 Mar. 18 Mar. 18 Apr. 19 Mar. 28 Common summer resident; occasionally winters.

339. Red-shouldered Hawk Buteo lineatus lineatus

May 17 Apr. 5 Jan. 1 Apr. 16 Mar. 10 May 9

Mar. 15

Common summer resident; sometimes wintering.

343. Broad-winged Hawk Buteo platyperus

Apr. 6 Feb. 27 Apr. 29 Apr. 22 Apr. 29 Apr. 19 Mar. 15

Fairly common migrant; a few breed.

347a. Rough-legged Hawk

Apr. 3

Archibuteo lagopus sancti-johannis

Rather rare winter visitant.

357. Pigeon Hawk Mar. 24

May $3 \quad$ May $6 \quad$ Apr. $19 \quad \ldots . . . . . . . \quad$ Mar. 24

Rare summer resident, commoner during migraticns.

360. Sparrow Hawk

Faico sparverius sparverius

Jan. 18 Mar. 14 Mar. 10 Mar. 18 Mar. 15 Mar. 16 Mar. 19 Mar. 14

Fairly common summer resident; sometimes wintering.

364 . Osprey

Pandion haliaetus carolinensis

Apr. 29 May 25

Rare visitant. Mr. Coale has a specimen taken at Highland Park.

366. Long-eared Owl

Asio wilsonianus

Mar. 31 Apr. 10 Feb. 13

Apr. 24

Rather uncommon summer resident; sometimes wintering.

367. Short-eared Owl

Asio flammeus

Jan. 16 Jan. 10

Rather rare permanent resident.

368. Barred Owl

Strix varia varia

Jan. 18

Feb. 24

Rare winter resident; formerly nested.

372. Saw-whet Owl

Cryptoglaux acadica acadica

\section{Apr. 8 Apr. 1}

Rare winter resident; is said to have summered.

373. Screech Owl

Otus asio asio

Feb. 12 Jan. 1 - Jan. 8 Feb. 28 Jan. $2 \quad$ Mar. 24 Mar. 23 Mar. 22

Common permanent resident.

387. Yellow-billed Cuckoo

Coccyzus americanus americanus

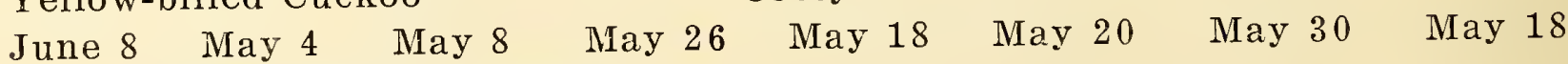
Common summer resident.

388. Black-billed Cuckoo Coccyzus erythrophthalmus

May 17 May $10 \quad$ May $13 \quad$ May $16 \quad$ May $18 \quad$ May $19 \quad$............. May 18 Common migrant; rather uncommon summer resident.

390. Belted Kingfisher Ceryle alcyon

Mar. 24 Jan. 2 Mar. $25 \quad$ Mar. $18 \quad$ Mar. 19 Mar. 17 Feb. 8 Mar. 24 Common summer resident; occasionally winters in open seasons. 


\begin{tabular}{lllllll}
\hline 1913 & 1914 & 1915 & 1916 & 1917 & 1918 & 1919
\end{tabular}

393. Fiairy Woodpezker Dryobatez villosus villosus

Jann. 1 Jan. 1 Jan. 1 Jan. 1 Jan. 1 Jan. 1 Fe's. 2 Feb. 21 Fairiy common permanent i esident.

$394 \mathrm{c}$. Downy Wcodpecker

Dryobates pubescens medianus

Jミn. 1 Jan. 1 Jan. 1 Jan. 1 Jan. $1 \quad$ Jan. $1 \quad$ Jan. 16 Fej. 17 Common permanent rexident.

400. Arctic Three-toed Woodpecker Picoides arcticus

Jan. 18

A very rare winter visitant. Has been taken by Mr. Stoddard at Miller.

402. Iellow-bellied Sapsucker

Sphyrapicus varius varius

Mar. 22 Apr. 4 Apr. 5 Mar. 24 Mar. 28 Mar. 31 Apr. 1 Mar. 24 Abundant migrant.

406. Red-keaded Woodpenker Melanerpes erythrocephalus

Feb. 15 Jan. 10 Apr. 13 Apr. 23 Jan. 1 Jan. 1 Apr. 22 Apr. 23 Common summer resident, often wintering.

409. Red-bellied Woodpecker Centurus carolinus

Miay 20

Very rare visitant. Mr. Coale records a breeding pair in Lincoln Park.

412a. Northern Flicker

Colaptes auratus luteus

Feb.9 Apr.2 Feb.9 Mar. 18 Jan. 1 Mar. 29 Mar. 23 Mar. 21 Ve:y common summer resident; sometimes wintering.

417. Whip-poor-will

Antrostromus vociferus pciferus

Apr. 24 Mar. 29 Apr. 25 May 1 May 18 May 4 May 3 Apr. $2 \%$

Not uncommon summer resident.

420. Nighthawk

Chordeiles virginianus virginianus

May $20 \quad$ May $11 \quad$ May $20 \quad$ May $10 \quad$ May $6 \quad$ May $13 \quad$ May $18 \quad$ May 10 Very common summer resident.

423. Chimney Swift

Chaetura pelagica

Apr. 23 Apr. 24 Apr. 26 Apr. 20 Apr. 18 Mray 3 Apr. 19 Apr. 21 Very common summer resident.

428. Ruby-throated Hummingbird Archilochus colubris

May 12 May 15 May 11 May 26 May 23 May 13 May $30 \quad$ May 23 Fairly common summer resident.

444. Kingbird

Tyrannus tyrannus

Apr. 26 Apr. 27 Apr. 27 Apr. 29 May 18 May 18 May 18 May 10 Fairly common summer resident.

452. Crested Flycatcher Myiarchus crinitus

$\begin{array}{llllllll}\text { May } 1 & \text { May } 3 & \text { May } 5 & \text { Apr. } 30 & \text { May } 16 & \text { May } 4 & \text { May } 6 & \text { May } 10\end{array}$ Rather rare summer resident; common during migrations.

456. Phoebe 'Sayornis phoebe

Mar. 24 Mar. 29 Apr. 5 -Mar. 23 Mar. 23 Mar. 18 Mar. 19 Mar. 24 Fairly common summer resident.

459. Olive-sided Flycatcher Nuttallornis borealis

May 21 May 19 May 11 May 8 May 29

Uncommon migrant. 


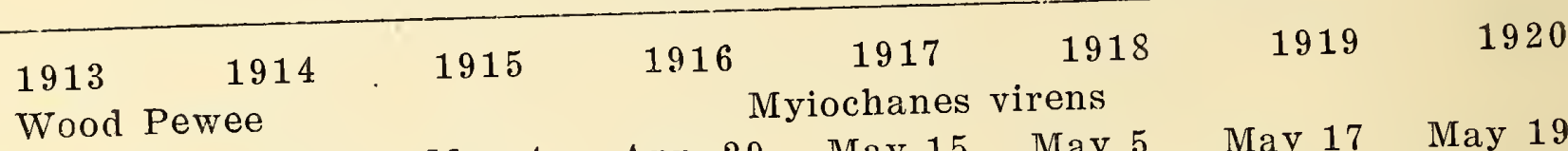

461. Wood Pewee

May 12 Apr. 27 May 4. Apr. $29 \quad$ May 15 May 5 May 17 May 19 Common summer resident.

463. Yellow-bellied Flycatcher $\begin{array}{lllllll}\text { May } 18 & \text { May } 17 & \text { May } 8 & \text { May } 17 & \text { May } 20 & \text { May } 28 & \text { May } 19\end{array}$ Fairly common migrant.

465. Acadian Flycatcher

Empidonax virescens

May 21 May 3 May 9 May 26 May 18 May 11 May 28 May 15

Rare summer resident; fairly common during migrations.

466. Traill's Flycatcher

May 22

Empidonax trailli trailli

May $19 \quad \ldots \ldots \ldots . . . . . . . . . . . . \quad$ May 19

Breeds commonly near Argo; rare elsewhere.

467. Least Flycatcher

Empidonax minimus

May 21 Apr. 28 May 4 May $6 \quad$ May $12 \quad$ May $4 \quad$ May 20 May 2

Common migrant; rather uncommon summer resident.

474b. Prairie Horned Lark
Apr. 6
Feb. 20
Feb. 22
Fairly common permanent resident.

Otocoris alpestris praticola

477. Blue Jay

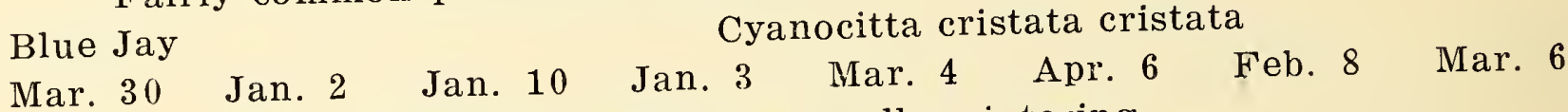
Very common summer resident generally wintering.

488. Crow

Corvus brachyrhynchos brachyrhynchos

Apr. 1 Mar. 14 Feb. 27 Jan. 22 Feb. 12 Mar. 18 Feb. 1 Feb. 22 Very common permanent resident.

494. Bobolink

Dolichonyx oryzivorus

Apr. 26 Apr. 26 Apr. 25 May 6 May 10 May 11 May 10 May 8 Common summer resident.

495. Cowbird

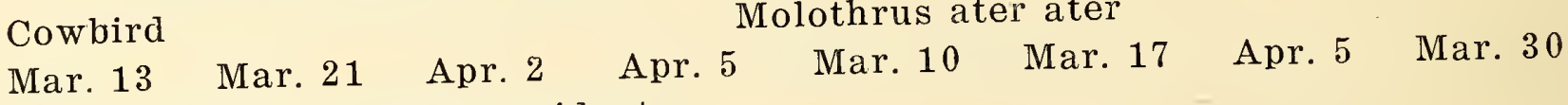

Abundant summer resident.

497. Yellow-headed Blackbird Xanthocephalus xanthocephalus

Apr. 24

Formerly common summer resident, but becoming rarer. The only record

for a breeding colony in recent years in our area is a colony which nests at

Hyde Lake, where in 1920 we found about twenty-five pairs.

498. Red-winged Blackbird Agelaius phoeniceus phoeniceus

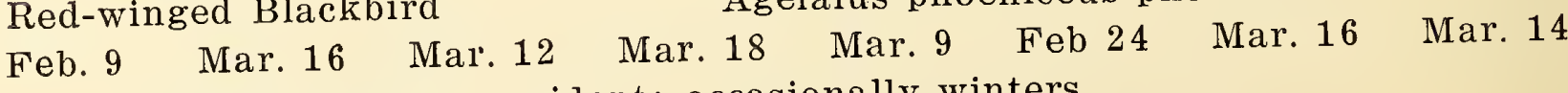

Abundant summer resident; occasionally winters.

501c. Southern Meadowlark Sturnella magna argutula

Mar. 12 Mar. 14 Feb. 27 Mar. 18 Mar. 9 Mar. 13 Mar. 16 Mar. 13

Abundant summer resident; sometimes winters during mild seasons.

506. Orchard Oriole

Icterus spurius

May 9 May 10

Rather rare summer resident.

507. Baltimore Oriole

Ict $₫$ rus galbula

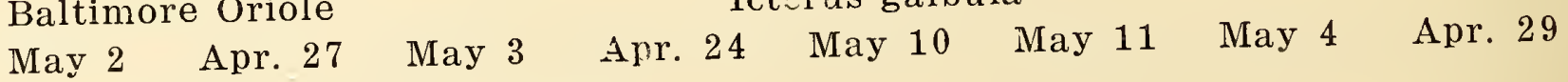
Common summer resident. 


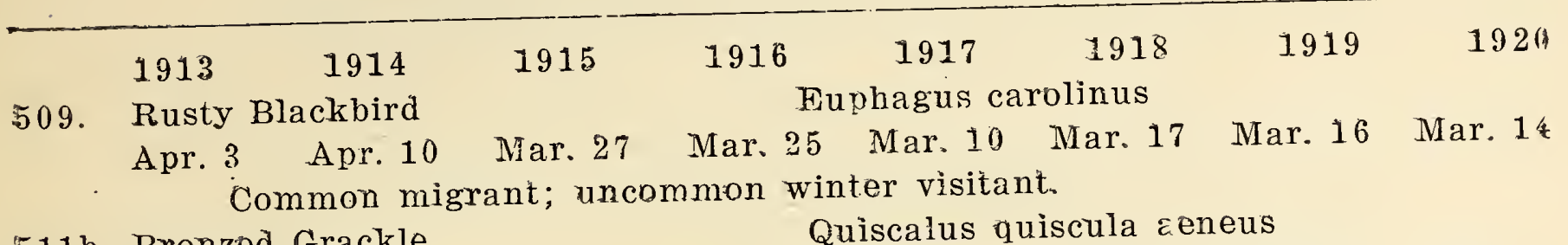

511 b. Bronzed.Grackle Quiscalus quiscula ceneus

Mar. 14 Mar. 14 Jan. 1 Mar. 13 Mar. 10 Mar. 5 Mar. 13 Mar. 13 Abundant summer resident; only two winter records.

517. Purple Finch

Carpodacus purpureus purpureus

Mar. 29 Mar 2 Apr. 8 Apr. 14 Jan. 1 May $25 \quad \ldots \ldots \ldots . . .$. Apr. 17 Rather common migrant; a few winter.

521. Crossbili

Loxia curvirostra minor

Feb. 18

Very uncommon and irregular winter resident,sometimes remains till June

528. Redpoll

Jan. 29 Feb. 13

Common but irregular winter resident.
Acanthìs linarìa linaria

Feb. 18

Mar. 6

52 9. Goldfinch

Astragalinus tristis trìstis

May 2 Jan.16 - Feb. 20 Mar. 29 Mar. 24 May 11 Apr. 26 Mar. 29

Common permanent resident.

533. Pine Siskin

$$
\text { Apr. } 29 \text { May } 4 \quad \ldots . . . . . . \quad \text { Apr. } 1
$$

Spinus pinus

Fairly common winter visitant, most common during May.

534. Snowflake

Plectrophenax nivalis nivalis

Feb. 22

Irregular winter visitant.

536. Lapland Longspur

Mar. 1 Jan. 3 Jan. 1

Calcarius lapponicus lapponicus

Common winter visitent.

537. Smith's Longspur

Calcarius pictus

Apr. 19

Rare and very irregular migrant.

*** English Sparrow

Passer hostilis

Jan. 1 Jan. 1 Jan. 1 Jan. 1 Jan. 1 Jan. 1 Jañ. 1 Jan. 1 Very abundant permanent resident.

540. Vesper Sparrow

Pooecetes gramineus graminèus

Apr. 5 Apr. 9 Apr. 5 Apr. 1 Mar. 25 Apr. 13 Apr. 19 Mar. 28 Fairly common summer resident.

542a. Savanna Sparrow

Passerculus sándwichensìs saavañnà

Apr. 20 Mar. 29 Apr. $6 \quad$ Mar. 19 Apr. $11 \quad \ldots \ldots \ldots$ May 3 Apr. 24 Common migrant; a few breed.

546. Grasshopper Sparrow

Ammodramús savannarum áustralís Apr. 28 Apr. 15 Apr. $13 \quad$ Apr. $26 \quad$ Apr. 25 Apr. 19 Mar. 28 Common summer resident.

547. Henslow's Sparrow

Passêrherbulus henslowi hênslowi

Apr. 22 Apr. 18 Apr. 18 Apr. 17 Apr. 18

Apr. 19 Maý 10 Uncommon summer resident. . 


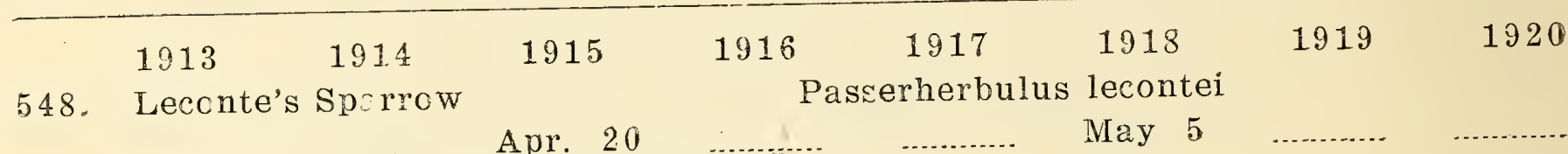

Rare migrant; one breeding rezord.

552. Lark Sparrow

Chondestes grammacus grammacus

Apr. 26

Apr. 17

Apr. 26

Fairly rare summer resident.

554. White-crowned Sparrow Zonotrichia leucophrys leucophrys

May 2 May 1 May 4 Apr. 30 May 8 May 4 May. 4 May 9 Common migrant, but not nearly so abundant as albicollis.

558. White-throated Sparrow Zonotrichia alhicollis

Apr. 22 Apr. 18 Apr. 15 Apr. 16 Apr. 18 Apr. 13 Apr. 7 Mar. 24 Abundant migrant.

559. Tree Sparrow

Spizella monticola monticola

Jan. 1 Jan. 1 Jan. 1 Feb. 22 Jan. 1 Jan. 1 Mar. 16 Mar. 6 Common winter resident.

560. Chipping Sparrow Spizella passerina passerina

Mar. 20 Apr. 8 Apr. 5 Apr. 11 Apr.11 Mar. 28 Apr. 19 Mar.28 Common migrant; breeds commonly in restricted areas.

561. Clay-colcred Sparrow Spizella pallida

May 8

Accidental straggler from the west; only about three rezords.

563. Field Sparrow

Spizella pusilla pusilla

Apr.6 Apr. 10. Apr. 7 Apr. 12 Mar. 29 Apr. 7 Mar. 23 Mar. 20 Common summer resident. Arrives esrlier in the spring than records indicate

567. Slate-colored Junco Junco hyemalis hyemalis

Jan. 18 Jan. 2 Jan. 2 Jan. 23 Jan. 1 Jan. 1 Jan. 25 Mar. 15 Fairly common winter resident; abundant in the spring and fall. During cold springs they are often seen until the middle of May.

575a. Bachman's Sparrow

Percaea aestivalis bachmani

\section{June 13}

"Since their appearance here in River Forest, May 9, 1915, when I saw them until July 1, I have seen no more. Dr. Lewy howerer, saw one in Jackson Park, June 1918."-Eifrig. They nested in 1916 in an oak wood north of La Grange. There is a spring date for 1920 in River Forest.

581. Song Sparrow

Melospiza melodia melodia

Jan. 18 Jan. 16 Jan. 11 Mar. 18 Feb. 4 Jan. 1 Mar. 16 Mar. 14 Very common summer resident; fairly common winter resident.

583. Lincoln's Sparrow Melospiza lincolni lincolni

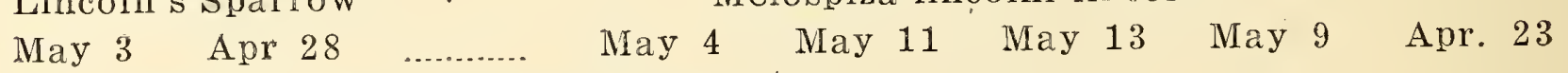
Fairly common migrant;-rare summer resident.

584. Swamp Sparrow

Melospiza georgiana

Apr. 20 Apr. 11 Apr. 9 Apr. $1 \quad$ Mar. 30 Apr. 25 Apr. 7 Mar. 25

Common summer resident; remarkable scarcity west of Chicago in summer

585. Fox Sparrow

Passerella iliaca iliaca

Mar. 13 Mar. 1 Apr. 5 $\quad$ Mar. 25 Mar. 11 Mar. 5 Mar. 16 Mar. 15 Very common spring and fall migrant. 


$\begin{array}{lllllll}1913 & 1914 & 1915 & 1916 & 1917 & 1918 & 1919\end{array}$

587 . Towhee Pipilo erythrophthalmus erythrophthalmus

Mar. 20 Mar. 28 Apr. $6 \quad$ Mar. 25 Mar. 23 Mar. 17 Mar. 18 Mar. 14 Common summer resident; very common during the migrations.

593. Cardinal

Cardinalis cardinalis cardinali $\mathrm{s}$

Feb. 15 Jan. 1 Jan. 1 Jan. 1 Jan. 1 Jan. $1 \quad$ Jan. 25 Mar. 6 A fairly common permanent resident, especially along the Desplaines River, and in the Dune region

595. Rose-breasted Grosbeak Zamelodia ludoviciana

$\begin{array}{llllllllll}\text { May } 3 & \text { Apr. } 28 & \text { May } 4 & \text { Apr. } 29 & \text { May } 10 & \text { May } 7 & \text { May } 7 & \text { May } 10\end{array}$

A fairly common summer resident; common migrant.

598. Indigo Bunting Passerina cyanea

May 10 May 5 May 5 May $11 \quad$ May $19 \quad$ Mía $13 \quad$ May $17 \quad$ May 19 Common summer resident.

604. Dickcissel Spiza americana

May $15 \quad$ May $31 \quad$ May 31

May 18

May 10

Formerly an abundant summer resident, but in the decrease in numbers.

608. Scarlet Tanager

Piranga erythromelas

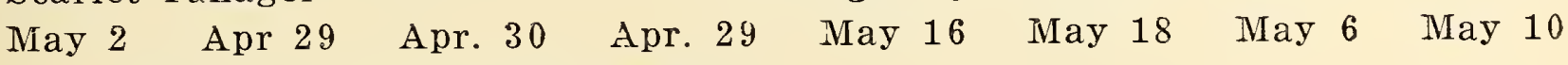

Fairly common summer resident.

611. Purple Martin

Progne subis subis

Apr. 28 Apr. $21 \quad$ Apr. 9 Apr. 10 Apr. $14 \quad$ Apr. 23 Apr. 10 Mar. 27

Very common summer resident.

612. Cliff Swallow

Petrochelidon lunifrons lunifrons

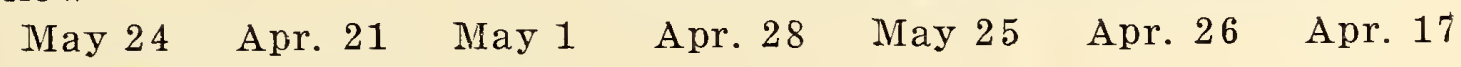
Fairly common summer resident

613. Barn Swallow

Hirundo erythrogastra

Apr. 19 Apr. 19 Apr. 11 Apr. 17 Apr. 14 Apr. 28 Apr. 18 Apr. 21 Very common summer resident.

614. Tree Swallow

Iridoprocne bìcolor

Apr. 2 Apr. 17 Apr. 8 Mar. 23 Mar. 31 Apr. 23 Apr. 5 Apr. 10 Uncommon summer resident; more common during migrations.

616. Bank Swallow

Riparia riparia

Apr. 24 Apr. 4 Apr 19 Apr. 20 May 11 May 2 Apr. 24 Common summer resident.

617. Rough-winged Swallow

Stelgidopteryx serripennis

May 28 May 1 May 2

Rare migrant; breeds sparingly.

618. Bohemian Waxwing Bombycilla garrula

May 22

Irregular winter visitant; a flock of three hundred observed at Jackson Park Nov. 27, 1919.' Abundant in the winter 1919-1920.

619. Cedar Waxwing

Bombycilla cedrorum

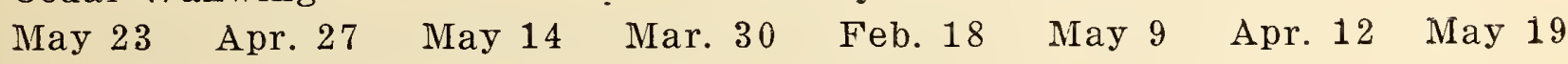
Common summer resident; occasionally wintering.

621. Northern Shrike

Lanius borealis

Mar. 15 Apr. 4 Feb. 8 Mar. 5

Uncommon winter resident. 


$1913 \quad 1914 \quad 1915 \quad 1916 \quad 1917 \quad 1918 \quad 1919$

1920

622e. Migrant Shrike Lanius ludovicianus migrans

Mar. 27 Apr. 4 Apr.6 Mar. 16 Apr. 15 Mar. 16 Apr.5

Fairly common summer resident.

624. Red-eyed Vireo Vireosylva olivacea

$\begin{array}{lllllll}\text { May } 2 & \text { Apr. } 29 & \text { May } 5 & \text { Apr. } 29 & \text { May } 16 & \text { May } 5 & \text { May } 17 \\ \text { May } 10\end{array}$ Common summes resident.

626. Philadelphia Vireo Vireosylva philadelphica

May 30

Miay 19

$\mathrm{MI} \approx \mathrm{y} \cdot 22$

Rather rare migrant.

627. Warbling Vireo

Vireosylva gilva gilva

Apr. 30 Apr. 27 May 9.. May $11 \quad$ May 16

May 19

Uncommon summer rexident.

628. Yellow-throated Vireo Lanivireo flavifrons

May 3 Apr. 26 May 15 Apr 30 May 29

Rather uncommon migrant; rarely breeds.

629. Blue-headed Vireo Lanivireo solitarius sclitarius

May 17 May 3 May 9 Apr 30 May 16 May 4 May 7 May 11 Rather uncommon migrant.

636. Black and White Warbler Mniotilta varia

Apr. 24 Apr. 18 Apr. 26 Apr. 19 May 3. May 5 May 4 Apr. 22 Common migrant. Three were seen July 4, 1914 just north of Riverside on the Des Plaines river. They probably bred in the vicinity.

637. Prothonotary Warbler Protonataria citrea
May 1
Apr. 27 Apr. 24
Apr. 29 May $11 \quad$ May 4
$\operatorname{May} 1$ Fairly common migrant.

\$41. Blue-winged Warbler

Vermivera pinus

May 16

Rare migrant; mo:e common in the fall; may breed.

342. Golden-winged Warbler

Vermivora chrysoptera

$\begin{array}{llllllll}\text { May } 3 & \text { Apr. } 28 & \text { May } 3 & \text { May } 6 & \text { May } 16 & \ldots \ldots \ldots . . . . & \text { May } 7 & \text { May } 9\end{array}$ Fairly common migrant.

645. Nashville Warbler

Vermivora rubricapilla rubricapilla

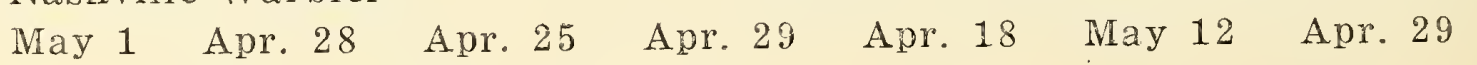

Common migranc.

646. Orange-crowned Warbler May 15 May 4

Uncommon migiant.

647. Tennessee Warbler

May 3 May 6 May 1 Fairly common migrant.

648a. Northern Parula Warbler

Vermivora celata celata Apr. 23 May $2 \quad$........... May 7 May 15

Vermivora peregrina May 16 May 3 May 20 May 19

Compsothlypis americana usneae

$\begin{array}{lllllll}\text { Apr. } 26 & \text { Apr. } 29 & \text { Apr. } 27 & \text { Apr. } 30 & \text { May } 15 & \text { May } 4 & \text { May } 17 \\ \text { May } 19\end{array}$ Rather uncommon migrant.

650. Cape May Warbler Dendroica tigrina

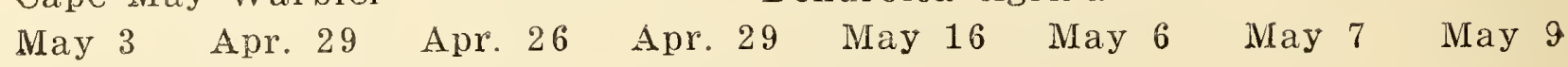
Common migrant, on the increase in numbers. 


$\begin{array}{lllllll}1913 & 1914 & 1915 & 1916 & 1917 & 1918 & 1919\end{array}$

652. Yellow Warbier Dendroica aestiva ae

May 2 Apr. 25 Apr. 25 Apr. 29 May 12 May $6 \quad$ May 3 May 6 Abundant summer resident.

654. Black-throated Blue Warbler Dendroica caerulescens caerulezcens

May 2 May 3 May 3 May 5 May 10. May 5 May 6 May " Common migrant.

655. Myrtle Warbler Dendroica coronata

Apr. 18 Apr. 6 Apr. $10 \quad$ Apr. $10 \quad$ Apr. 11 Apr. 22 Apr. 5 Apr. 11 Abundant migrant.

£57. Magnolia Warbler Dendroica magnolia

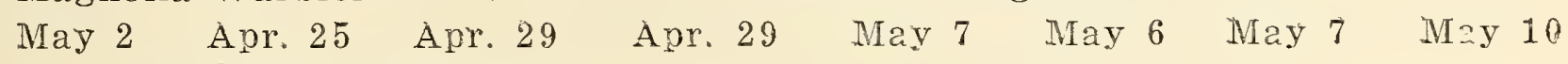

Very common migrant.

658. Cerulean Warbler Dendroica cerulea

May $1 \quad$........... May 4 May $7 \quad \ldots . . . . . . . \quad$ May 14

Rare summer resident.

659. Chestnut-sided Warbler Dendroica pensylvanica

May 3 Apr. 28 May 3 May 5 May 16 . May 4 May 4 May 9 Common migrant; a few breed.

660. Bay-breasted Warbier Dendroica castanea

May 2. May 15 Apr. 27 May 7 May 16 May 16 May 17 May 18 Fairly common migrant.

661. Blackpoll Werbler Dendroica striata

$\begin{array}{llllllll}\text { May } 5 & \text { Apr. } 28 & \text { May } 12 & \text { May } 11 & \text { May } 18 & \text { May } 15 & \text { May } 17 & \text { May } 18\end{array}$ Fairly common migrant.

662. Blackburnian Warbler, Dendroica fusca

May 1 Apr. 28 May 3 Apr. 29 May 15 May 6 May 4 May 6 Common migrant.

A male of this species was observed, July 11th, 1920 , in a pine clearing between Dune Park and Mineral Springs, Ind. The bird was in full song, which would indicate that it was nesting.

667. Black-throated Green Warbler Dendroica virens

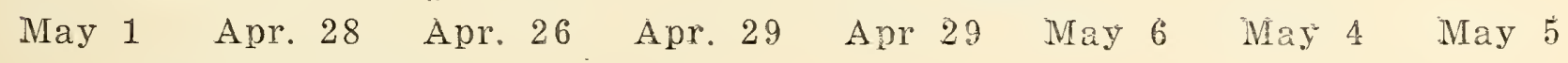
Common migrant.

671. Pine Warbler Dendroìca vìgorsi

Apr. 22 Apr. 25 Apr. 20 May 3 Apr. 18 Apr. 27 May 4 May 10 Rather uncommon migrant.

672. Palm Warbler

Dendroica paimarum

Apr. 22 Apr. 21 Apr. 20 Apr. 10 Apr. 18 May 2 Apr. 18 Apr. 30 Abundant migrant.

672. Praỉie Warbler Dendroỉca discolor

May 4 ..................... May 12 May 11 May 6

Most writers consider this a very rare migrant We have seen it about eight times.

674. Ovenbird

Seiurus aurócapillus

May 1 Apr. 28, May 4 Apr. 30 May 10 May 5 May 4 May 2 Common migrant; uncommon summer resident. 


$\begin{array}{lllllll}1913 & 1914 & 1915 & 1916 & 1917 & 1918 & 1919\end{array}$

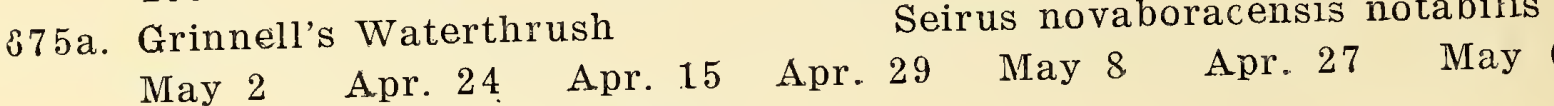

Very common migrant.

676. Louisiana Waterthrush

May $15 \quad$ May 13

Rare summer resident.

677. Kentucky Warbler

May 16

Seiurus motacilla

May 18

May 13

Opororuis formosus

June 2

Oporornis agilis
Rare visitant from the south.

678. Connecticut Warbler

May 16 May 4 May 18 May 6 May 18 May 22
Tolerably common migrant.

679. Mourning Warbler
May 17 May 11 Fairly common migrant.

681: Maryland Yellowthroat Geothlypis trichas trichas

May 11 Apr. 28 May 8 May 2 May 11 May 7 May 4 May 2 Common summer resident.

683. Yellow-breasted Chat

Icteria virens virens

May 18

Uncommon summer resident.

685. Wilson's Warbler Wilsonia pusilla pusilla

May 17 May. 10 May 12 May 12 May 1.10 May 11 May 17 May 10

Fairly common migrant.

686. Canada Warbler Wilsonia canadensis

May 15 May 10 May 14 May 7 May 16 May 15 May 18 May 10

Fairly common migrant.

687. Redst $=$ rt

Setophaga ruticilla

May 2 Apr. 27 Apr. 25 May 6 May 14 May 3 May 17 May 6

Abundant migrant; not uncommon summer resident.

704. Catbird

Dumetella carolinensis

May 3 Apr.27 Apr. 29 Apr. 29 May 11 May 12 May 4 Apr. 29

Common summer resident.

705. Brown Thrasher

Apr. 19 Apr. 15 Apr 18

Common summer resident; one record for the winter.

718. Carolina Wren

Thryothorus ludovicianus ludovicianus

Mar. 24 Jan. 1 Mar. 31

Uncommon permanent resident, on the increase in numbers.

719. Bewick's Wren

Thryomanes bewicki bewicki

Very rare visitant. Nelson reports one.

721. House Wren

Troglodytes aedon aedon

Apr. 25 Apr. 2 F'eb. 22 Apr. 29 Mar. 31 Apr. 25 May 3 Mar. 24

Common summer resident. 


$\begin{array}{lllllll}1913 & 1914 & 1915 & 1916 & 1917 & 1918 & 1919\end{array}$

722. Winter Wren Nannus hiemalis hiemalis

Mar. 29 Apr. $10 \quad$ Apr. $5 \quad$ Apr. $4 \quad$ Mar. 30 Apr $6 \quad$ Apr. 13 Apr. 17 Fairly common migrant

724. Short-billed Marsh Wren Cistothorus stellaris

May $10 \quad$............ May 7

Fairly common summer resident in restricted areas.

725. Long-billed Marsh Wren Telmatodytes palustris palustris $\begin{array}{lllllll}\text { Apr. } 27 & \text { May } 8 & \text { Apr. } 20 & \text { Apr. } 18 & \text { May } 8 & \text { May } 7 & \text { May } 1\end{array}$ Very common summer resident.

726. Brown Creeper Certhia familiaris americana

Jan. 26 Jan. 1 Jan. 1 Mar. 30 Mar. $4 \quad$ Feb. 24 Jan. 17 Mar. 31 Common winter resident; commoner in migrations.

727. White-breasted Nuthatch Sitta carolinensis carolinensis

Jan. 2 Jan. 1 Jan. 1 Jan. $3 \quad$ Jan. $1 \quad$........... Jan. 17 Mar. 27 Fairly common permanent resident.

728. Red-breasted Nuthatch Sitta canadensis

$\begin{array}{llllllll}\text { Jan. } 4 & \text { Apr. } 25 & \text { Apr. } 26 & \text { Mar. } 18 & \text { Feb. } 15 & \text {........... } & \text { Jan. } 1 & \text { Mar. } 6\end{array}$ Fairly common migrant; sometimes winters and rarely breeds

731. Tufted Titmouse

May 10 June 20

Baeolophus bicolor

Uncommon permanent resident.

735. Chickadee

Penthestes atricapillus atricapillus

Feb. 9 Jan. 1 Jan. 1 Jan. 2 Jan. 1

Feb. 8 Feb. 22 Fairly common permanent resident.

748. Golden-crowned Kinglet Regulus satrapa satrapa

Mar. 18 Jan. 10 Jan. $3 \quad$ Mar. 26 Mar. 30 Apr. 4 Apr. 5 Mar. 24 Common migrant; irregular winter visitant.

749. Ruby-crowred Kinglet

Regulus calendula calendula

Mar. 29 Apr. 6 Apr. 5 Apr. $1 \quad$ Mar. $30 \quad$ Apr. 27 Apr. 5 Mar. 21

Very common migrant.

751. Blue-gray Gnatcatcher Polioptila caerulea caerulea

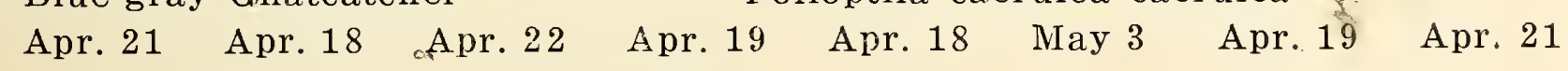
Uncommon suinmer resident.

755. Wood Trush

Hylocichla mustelina

Apr. 23 Apr. $24 \quad$ May $3 \quad$ Apr. 29 $\quad$ May $13 \quad$ May $11 \quad$ May 17 May \& Common summer resident.

756. Veery

Hylocichla fuscescens fuscescens

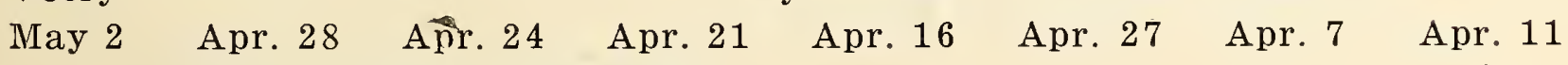
Common migrant; may breed.

757. Gray-cheeked Thrush

Hylocichla aliciae aliciae

Apr. 26 Apr. 30 Apr. 26 May 6 Má 16 May 4 May 5 May 10 Fairly common migrant.

758a. Olive-backed 'Thrush

Hylocchla ustulata swainsoni

Apr. 26 Apr. 22 Apr. 24
Very common migrant.

Apr. 20 May $5 \quad$ May 4 May $5 \quad$ Apr. 22 


$\begin{array}{llllllll}1913 & 1914 & 1915 & 1916 & 1917 & 1918 & 1919 & 1920\end{array}$

$759 \mathrm{~b}$. Hermit Thrush Hylocichla guttata pallasi

Apr. 3 Mar. 29 Apr. 5 Mar. $25 \quad$ Mar. $30 \quad$ Apr. 4 Apr. $5 \quad$ Mar. 27 Very common migrant.

761. Robin

Planesticus migratorius migratorius

Jan. 18 Mar. 14 Feb. 13 Jan. 2 Feb. 25 Feb. 24 Jan. 16 Mar. 14 Abundant summer resident; a few winter each year.

766. Bluebird

Sialia sialis sialis

Mar. 13 Mar. 15 Feb. 13 Feb. 22 Mar. $10 \quad$ Feb. $24 \quad$ Mar. 19 Mar. 14 Common summer resident.

\section{APPENDIX}

The following are birds observed only in the fall migration.

133. Black Duck

Anas rubripes

Dec. 13

Uncommon migrant, and•winter visitant.

135. Gadwall

Chaulelasmus streperus

Dec. 11

Uncommon migrant; occasionally winters.

258a. Western Willet

Uncommon migrant

270. Black-bellied Plover

Aug. 18

Formerly common, now rather rare migrant. Aug. 5 Aug. 31

514. Evening Grosbeak

Catoptrophorus semipalmatus inornatus

Squatarola squatarola

Hesperiphona vespertina vespertina

Nov. 15

Rare and irregular winter visitant; common winter 1919-1920.

The following are subspecies whose occurrance is doubtful.

148. Scaup Duck (Marila marila). The Lesser Scaup Duck (Marila affinis) is decidedly the more common bird, but the large duck undoubtedly occurs in the winter. As positive identification without actual taking of specimens is difficult, this species cannot be positively included in the list.

474. Horned Lark (Otocoris alpestris alpestris). There are undoubtedly winter records of this bird in our area. It's subspecies, praticola, is a fairly common permanent resident.

675. Waterthrush (Seiurus novaboracensis novaboracensis). In all probability both forms of the Waterthrush migrate through the area, novaboracensis in the early spring, notabilis later. The Grinnel's Waterthrush however, is much commoner.

725 d. Prairie Marsh Wren (Telmatodytes palustris iliacus). This form does occur, according to Dr. Cory, but palustris is much the commoner.

756a. Willow Thrush (Hylocichla fuscescens salicicola). This bird is probably fully as common as fuscescens, but they are practically indistinguishable. 
.

. 

